\title{
Admission Systolic Blood Pressure Predicts Post-Operative Delirium of Acute Aortic Dissection Patients in the Intensive Care Unit
}

\author{
Zuli Fu (D) ${ }^{1,2}$ \\ Qian Xu' \\ Chiyuan Zhangl,3 \\ Hui Bai ${ }^{1}$ \\ Xuliang Chen' \\ Yanfeng Zhang' \\ Wanjun Luo' \\ Guoqiang Lin' \\ 'Department of Cardiovascular Surgery, \\ Xiangya Hospital, Central South \\ University, Changsha, Hunan, People's \\ Republic of China; ${ }^{2}$ Department of \\ Cardiovascular Medicine, Xiangya \\ Hospital, Central South University, \\ Changsha, Hunan, People's Republic of \\ China; ${ }^{3}$ Department of Cardiovascular \\ Medicine, The Third Xiangya Hospital, \\ Central South University, Changsha, \\ Hunan, People's Republic of China
}

Correspondence: Guoqiang Lin Department of Cardiovascular Surgery, Xiangya Hospital, Central South University, Changsha, 410008, People's Republic of China

$\mathrm{Tel} / \mathrm{Fax}+86-73 \mathrm{I}-84327097$

Email lgq186snoopy@126.com
Purpose: Post-operative delirium (POD) is a common complication after aortic surgery with poor outcomes. Blood pressure may play a role in the occurrence of POD. The study aimed to identify whether admission systolic blood pressure (SBP) level in the intensive care unit (ICU) is correlated with POD in acute Stanford type A aortic dissection (AAAD) patients undergoing aortic surgery.

Patients and Methods: We conducted a single-center retrospective cohort study enrolling consecutive 205 patients with acute type A aortic dissection undergoing aortic surgery. Patients were divided into 3 groups: low, normal, and high SBP level group. Outcomes of interest were POD, 30-day mortality and other complications including acute kidney injury, cardiac complications, spinal cord ischemia, stroke, and pneumonia. Confusion Assessment Method for Intensive Care Unit (CAM-ICU) method was used to assess POD. Univariate and multivariate logistic regression, Cox regression, and subgroup analysis were performed to uncover the association between SBP and POD.

Results: The mean age of these patients was $51 \pm 16$ years old. Thirty-six patients $(17.6 \%)$ developed POD. Patients with high admission SBP were more likely to develop POD (P < 0.01). Univariate analysis showed that high admission SBP was associated with a higher risk of POD among AAAD patients (OR, 3.514; 95\% CI, 1.478-8.537, P < 0.01). Multivariate logistic regression model confirmed that high SBP was an independent predictor of POD. Subgroup analysis indicated that patients with anemia and high admission SBP were at higher risk of POD.

Conclusion: High admission SBP was positively associated with the incidence of POD in AAAD patients who underwent surgical repair in ICU.

Keywords: acute Stanford type A aortic dissection, intensive care unit, major surgery, postoperative delirium, systolic blood pressure

\section{Introduction}

Acute Stanford type A aortic dissection (AAAD) is one of the most severe and lifethreatening cardiovascular diseases characterized by an urgent aortic dissection involving the ascending aorta and may be extended into the arch and descending aorta, which has a high mortality rate and generally requires emergent surgical intervention. ${ }^{1}$ Postoperative delirium (POD), triggered by multiple causes including acute medical illness, drug use or withdrawal, trauma, or surgery, is one of the major postoperative neurological complications of AAAD, defined as inattention, disorientation, and fluctuating consciousness, occurring within 30 days after surgery. ${ }^{2,3}$ Compared to other cardiac 
surgeries, AAAD patients might have a greater risk of POD, which leads to higher in-hospital and 6-month mortality, functional decline, greater rates of institutional discharge, longer lengths of stay (LOS), increased use of hospital resources, and higher health-care costs. ${ }^{3}$ Perioperative interventions are the best management for POD, for which the risk factors of POD in AAAD are needed to be identified.

High blood pressure is an independent risk factor of the rupture of aortic dissection, in-hospital mortality in AAAD patients, as well as postoperative complications such as delirium, myocardial infarction, stroke, and atrial fibrillation. ${ }^{4-7}$ Since the brain is sensitive to the changes in blood oxygen level which is adjusted by the contraction and relaxation of blood vessels, it has been indicated that blood pressure may play a role in cognitive disorder and POD. ${ }^{8}$ However, the association between blood pressure and POD in patients with AAAD in ICU remains unknown. Since the control of SBP in the management of AAAD is essential, the aim of this study, therefore, was to further identify whether admission SBP level in ICU is associated with POD in AAAD patients.

\section{Patients and Methods}

\section{Participants}

We consecutively collected 302 patients diagnosed with AAAD between January 2017 and December 2019 at Cardiovascular surgery department, Xiangya Hospital, Central South University in China. Sixty-seven patients were excluded because of death before surgery or refusing surgery. Twenty-five patients with preoperative delirium and 5 patients with censored data were excluded. There were 205 patients who underwent aortic surgery enrolled in analysis eventually (Figure 1).

\section{Anesthesia}

All patients were operated on under combined intravenousinhalation anesthesia and cardiopulmonary bypass. Deep hypothermic circulatory arrest (DHCA) and bilateral antegrade cerebral perfusion were performed with near-infrared spectroscopy (NIRS) monitoring the cerebral oxygen saturation. Regional cerebral cortical oxygen saturation $\left(\mathrm{rScO}_{2}\right)$ was maintained between $60 \% \sim 75 \%$. Anesthetic drugs

\section{2 adults, first-admitted patients were diagnosed as "AAAD"}

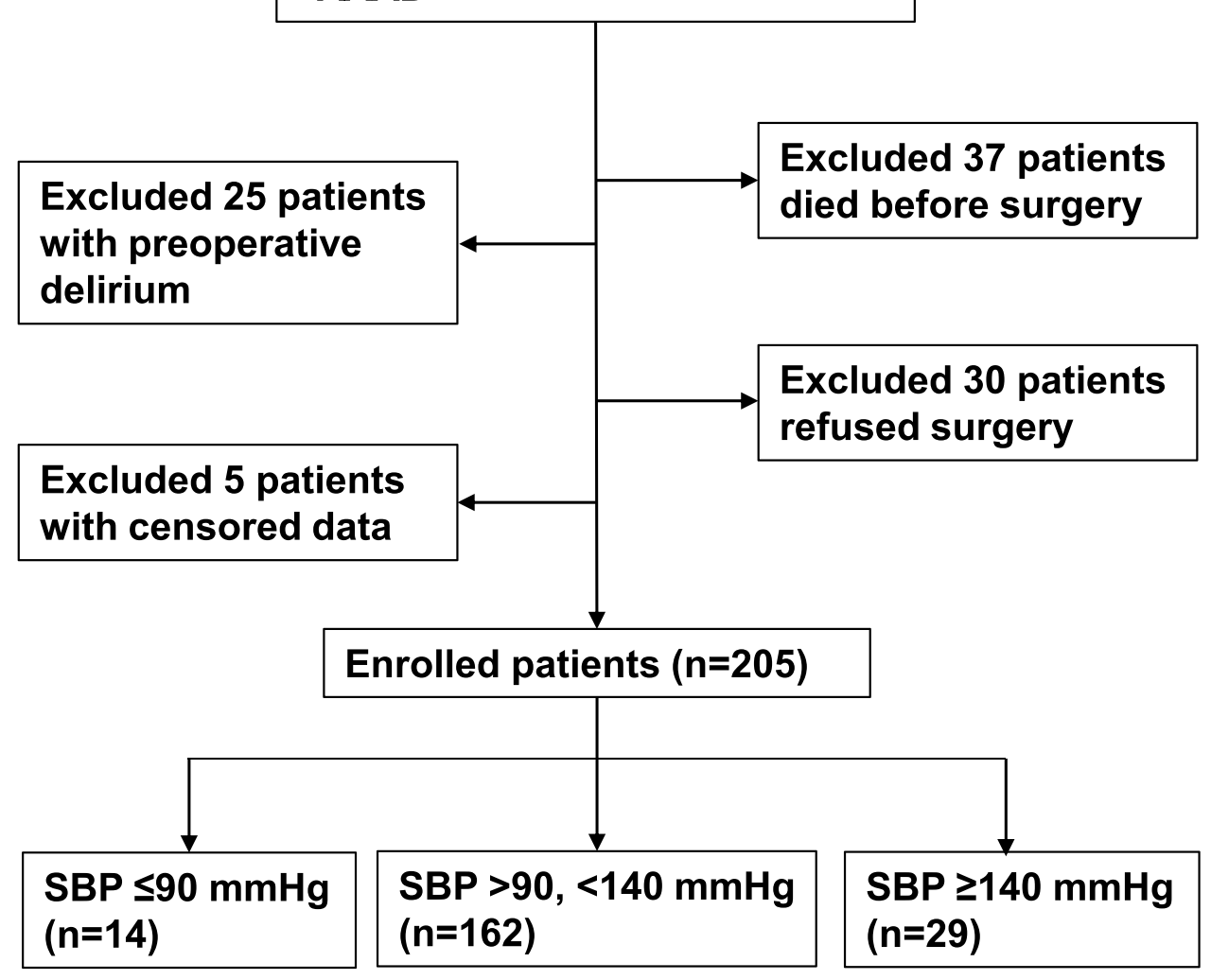

Figure I Flow chart of study population and groups. 
included midazolam, sufentanil, shunatracurium, etomidate, sevoflurane, and propofol. Intraoperative monitoring data included postapneic end-tidal carbon dioxide $\left(\mathrm{PetCO}_{2}\right)$, invasive arterial pressure, central venous pressure (CVP), pulmonary arterial pressure (PAP), cardiac output (CO), nasopharyngeal and rectal temperature, urine volume, arterial blood gas analysis (ABG), activated clotting time (ACT), and transesophageal echocardiography (TEE). Dexmedetomidine combining butorphanol was used for postoperative sedation and analgesia.

\section{Outcomes}

The primary outcome was POD. Thirty-day mortality and other postoperative complications including acute kidney injury, cardiac complications, spinal cord ischemia, postoperative stroke, and pneumonia were evaluated as second outcomes. POD assessment was performed 3 times a day $(7: 00,15: 00,23: 00)$ by ICU nurses who had received training in delirium assessment. Confusion Assessment Method for Intensive Care Unit (CAM-ICU) was used to evaluate and diagnose POD. The Richmond Agitation Sedation Scale (RASS) was used to determine the level of patients' consciousness. ${ }^{9}$ Patients with RASS $\geq-3$ were then assessed by CAM-ICU, including four features: (1) acute onset of changes in the course of mental status from the baseline or abnormal behavior fluctuation during the past 24 hours; (2) inattention evidenced by a score of $<8$ correct answers on either the visual or auditory components of the Attention Screening Examination; (3) disorganized thinking assessed by incorrect answers to $\geq 3$ questions and inability to follow the commands; (4) altered level of consciousness other than alert: vigilant, lethargic or in a stupor, or coma. Patients with features (1) and (2) and either feature (3) or (4) were identified as POD. 30day mortality was defined as death occurring within 30 days after surgery. Acute kidney injury was defined according to the KDIGO 2012 AKI criteria (1) an increase in serum creatinine ( $\mathrm{Scr}$ ) by $\geq 0.3 \mathrm{mg} / \mathrm{dl}$ within 48 hours, (2) an increase in Scr to $\geq 1.5$ times baseline occurred within the prior 7 days, (3) urine volume $<0.5 \mathrm{~mL} / \mathrm{kg} / \mathrm{h}$ for 6 hours. ${ }^{10}$ Cardiac complications included postoperative aortic insufficiency, acute myocardial infarction, acute heart failure, malignant arrhythmia, and cardiac tamponade. Spinal cord ischemia was diagnosed by newly onset paralysis, sensory loss, and urinary and bowel dysfunction. ${ }^{11}$ Postoperative stroke was defined as acute onset of focal neurologic deficit of cerebral origin persisting more than 24 hours without hemorrhage on computed tomography (CT) within 10 days after surgery. ${ }^{12}$ Pneumonia was defined as hospital-acquired pneumonia (pneumonia developing 48-72 hours after admission) or ventilator-associated pneumonia (pneumonia developing 48-72 hours after endotracheal intubation) occurring after the operation. ${ }^{13}$

\section{Clinical Data Collection}

Clinical variables of enrolled patients were obtained through a review of electronic medical records, which contained demographic information (gender and age), medical history including hypertension, coronary artery disease, cardiac valvular disease, diabetes, stroke/transient ischemic attack (TIA), chronic kidney disease, and Marfan's syndrome. Besides, all patients were free of alcohol or illicit drug addiction, or psychiatric history. Baseline information included blood pressure (systolic, diastolic, and mean arterial pressure) on ICU admission. Laboratory data included blood routine examination, hepatorenal function, coagulation function, and myocardial enzyme. Operation variables included surgical approaches, operation time, cardiopulmonary bypass time, aortic crossclamp time, and circulatory arrest time. Anemia was defined as hemoglobin $<120 \mathrm{~g} / \mathrm{L}$ in males and $<110 \mathrm{~g} / \mathrm{L}$ in females. Hyperuricemia was defined as uric acid $>428.0$ $\mu \mathrm{mol} / \mathrm{L}$ in males and $>357.0 \mu \mathrm{mol} / \mathrm{L}$ in females.

The outcomes included complications (delirium, acute kidney injury, cardiac complications, spinal cord ischemia, stroke, and pneumonia) and 30-day mortality. The definitions of all variables were based on the guidelines published by the Society of Thoracic Surgeons definitions. ${ }^{14}$ This study was carried out following the Declaration of Helsinki (World Medical Association 2013). ${ }^{15}$ We guarantee data confidentiality. Patient consent to review their medical records was waived for this study did not cause harm or risk to patients. The Ethics Committee of the Xiangya Hospital of Central South University approved the reporting of information obtained from our retrospective study (Ethical code: 202101003).

\section{Statistical Analysis}

Baseline characteristics of all patients were stratified to tertiles including low admission SBP level $(\leq 90 \mathrm{mmHg})$, normal admission SBP level (91-139mmHg), and high admission SBP level ( $\geq 140 \mathrm{mmHg}$ ). Normally distributed continuous data are presented as mean $\pm \mathrm{SD}$, whereas nonnormally distributed continuous data are presented as median (lower quartile-upper quartile). Categorical variables 
are expressed as the number of patients (\%). One-way ANOVA was used to analyze normally distributed continuous variables, and Mann-Whitney $U$-test was used to analyze non-normally and non-uniformed distributed continuous variables. Fisher's exact test or the chi-square test was used to analyze categorical variables.

We used adjusted multivariate models for 30-day POD incidence to facilitate clinical interpretation of our results. Variables based on epidemiological, medical history, clinical data, and laboratory tests were incorporated as potential confounders. In this model, covariates were adjusted to demographics, history of diabetes, coronary artery disease, cardiac valvular disease, Marfan's syndrome, chronic kidney injury, cardiopulmonary bypass time, aortic crossclamp time, hemoglobin, albumin, total bilirubin, alanine transaminase, creatinine, uric acid, and D-dimer.

Predictors of POD occurrence were identified using univariate logistic regression and included in a Cox proportional hazards model to determine predictors of delirium occurrence. Finally, subgroup analyses were conducted to evaluate the association between SBP and POD incidence in different subgroups, including age, gender, hypertension, anemia, hyperuricemia, urea nitrogen level, and creatinine level.

All statistical analyses were processed using IBM SPSS 23.0 software, and $P$-value $<0.05$ was considered statistically significant.

\section{Results}

\section{Characteristics of the Subjects}

A total of 205 patients were included in this study. The mean age of included patients was $51 \pm 16$ years, with 147 (71.7\%) males. The mean admission SBP was $120(95 \%$ CI, 109-134) mmHg. High admission SBP (SBP $\geq 140$ $\mathrm{mmHg}$ ) was present on admission in 29 (14.1\%), and low admission SBP (SBP $\leq 90 \mathrm{mmHg}$ ) was present on admission in $14(6.8 \%)$. Table 1 summarizes the differences in baseline characteristics between the 3 groups. The high SBP group had a higher rate of HBP history $(P=0.045)$, higher DBP $(P=0.007)$, and mean arterial BP $(P<0.001)$, whereas the low SBP group had a higher rate of Marfan's syndrome. Other medical histories, including diabetes, coronary artery disease, valvular disease, and chronic kidney disease were similar between the 3 groups. The laboratory examination data of the 3 groups were similar.

\section{Operative Characteristics}

Operative characteristics were compared between the 3 groups (Table 2). The rates of surgical approaches including Sun's, Sun's + root, ascending + arch, and Bentall were similar $(P=0.066)$. The average operation time was 7.6 (95\% CI, 6.5-9.0) hours, and CPB time was 187.0 (95\% CI, 166.5-210.5) minutes. Operation time, aortic cross-clamp time, CPB time, circulatory arrest time showed no significance between SBP groups.

\section{Outcomes}

POD and postoperative stroke were of statistical significance in 3 groups $(37.9 \%$ versus $7.1 \%$ and $14.8 \%$, $P=0.012 ; 0.6 \%, 7.1 \%$, and $13.8 \%, P=0.002)$. Thirtyday mortality, hospital stay, ICU stay, and other postoperative complications (acute kidney injury, cardiac complication, spinal cord ischemia, pneumonia) showed no significance between groups. These outcomes are summarized in Table 3.

\section{High Admission SBP is Directly Associated with the Incidence of POD in AAAD Patients with Surgical Repair}

Our initial logistic regression analysis results showed admission SBP level was positively associated with the incidence of POD (Table 4) (odds ratio [OR], 1.026 per $1 \mathrm{mmHg}$ increase; 95\% CI, 1.007-1.046; $P<0.01)$. To further explore the association between admission SBP level and POD, multivariate logistic regression analysis was conducted and listed in Table 5. Patients with high admission SBP had an increased risk of POD incidence (unadjusted OR, 3.514; 95\% CI, 1.478-8.357, $\mathrm{P}<0.05$ ).

In the multivariate logistic regression model (Table 5), after adjustment for demographics, history of diabetes, coronary artery disease, cardiac valvular disease, Marfan's syndrome, chronic kidney injury, cardiopulmonary bypass time, aortic cross-clamp time, hemoglobin, albumin, total bilirubin, alanine transaminase, creatinine, uric acid, and D-dimer, high admission SBP level remained a statistical predictor of POD in AAAD patients with surgical repair (adjusted OR, 4.655; 95\% CI, 1.708-12.687; P < 0.01). 
Table I Baseline Characteristics According to Admission Systolic Blood Pressure

\begin{tabular}{|c|c|c|c|c|c|}
\hline \multirow[t]{2}{*}{ Characteristics } & \multirow[t]{2}{*}{ Total $(n=205)$} & \multicolumn{3}{|c|}{ Blood Pressure (mmHg) } & \multirow[t]{2}{*}{$P$-value } \\
\hline & & $\leq 90(n=14)$ & $91-139(n=162)$ & $\geq 140(n=29)$ & \\
\hline \multicolumn{6}{|l|}{ Demographics } \\
\hline Gender & & & & & 0.258 \\
\hline Male & 147 (71.7\%) & $8(57.1 \%)$ & $120(74.1 \%)$ & 19 (65.5\%) & \\
\hline Female & $58(28.3 \%)$ & $6(42.9 \%)$ & $42(25.9 \%)$ & $10(34.5 \%)$ & \\
\hline Age, years & $51.0(44.0-57.0)$ & $50.8 \pm 15.5$ & $50.0(44.0-56.0)$ & $50.6 \pm 9.0$ & 0.589 \\
\hline \multicolumn{6}{|l|}{ Comorbidities } \\
\hline HBP & 149 (72.7\%) & $6(42.9 \%)$ & $122(75.3 \%)$ & $21(72.4 \%)$ & 0.045 \\
\hline Diabetes & II (5.4\%) & $0(0.0 \%)$ & $8(4.9 \%)$ & $3(10.3 \%)$ & 0.406 \\
\hline CAD & $15(7.3 \%)$ & $2(14.3 \%)$ & $10(6.2 \%)$ & $2(7.7 \%)$ & 0.332 \\
\hline Valvular disease & $15(7.3 \%)$ & $2(14.3 \%)$ & $10(6.2 \%)$ & $3(10.3 \%)$ & 0.332 \\
\hline Stroke/TIA & $9(4.4 \%)$ & I (7.l\%) & $5(3.1 \%)$ & $3(10.3 \%)$ & 0.397 \\
\hline CKD & $4(2.0 \%)$ & $0(0.0 \%)$ & $3(1.9 \%)$ & I (3.4\%) & 0.613 \\
\hline Marfan's syndrome & $10(4.9 \%)$ & $3(21.4 \%)$ & $6(3.7 \%)$ & I (3.4\%) & 0.031 \\
\hline Anemia & $98(48.0 \%)$ & $9(64.3 \%)$ & 71 (44.l\%) & $18(62.1 \%)$ & 0.092 \\
\hline \multicolumn{6}{|l|}{ Physical examination } \\
\hline DBP & $60.0(53.0-69.0)$ & $52.2 \pm 10.6$ & $60.6 \pm 11.3$ & $67.6 \pm 18.3$ & 0.007 \\
\hline Mean arterial pressure & $80.0(73.0-88.7)$ & $64.2 \pm 8.0$ & $79.9 \pm 9.6$ & $93.0(85.3-104.7)$ & 0.000 \\
\hline \multicolumn{6}{|l|}{$\begin{array}{l}\text { Admission laboratory } \\
\text { values }\end{array}$} \\
\hline WBC, K/uL & $11.6(8.9-14.4)$ & $12.1 \pm 3.3$ & $11.8(9.3-14.1)$ & $9.8(7.4-15.0)$ & 0.475 \\
\hline PLT, K/uL & $158.0(128.5-200.5)$ & $165.5(137.0-232.0)$ & $157.0(128.0-194.0)$ & $170.9 \pm 64.7$ & 0.471 \\
\hline $\mathrm{Hb}, \mathrm{g} / \mathrm{dL}$ & $129.0(\mid 14.0-140.5)$ & $131.2 \pm 20.7$ & $129.0(|14.0-14| .0)$ & $122.0(109.0-132.0)$ & 0.116 \\
\hline $\mathrm{TB}, \mu \mathrm{mol} / \mathrm{L}$ & $14.0(10.6-19.6)$ & II.4 (7.3-24.7) & $14.0(10.8-18.9)$ & $16.5 \pm 8.9$ & 0.482 \\
\hline ALT, U/L & $22.5(\mid 4.9-38.8)$ & $21.4(14.6-53.0)$ & $22.8(14.9-39.1)$ & $21.3(15.1-34.7)$ & 0.979 \\
\hline $\mathrm{BUN}, \mathrm{mmol} / \mathrm{L}$ & $6.9(5.2-8.9)$ & $6.7 \pm 2.6$ & $6.9(5.1-8.9)$ & $6.7(5.7-9.2)$ & 0.673 \\
\hline $\mathrm{Cr}, \mu \mathrm{mol} / \mathrm{L}$ & $104.9(84.3-134.2)$ & $99.0(89.1-128.8)$ & $105.1(86.1-134.4)$ & $98.0(76.0-147.5)$ & 0.811 \\
\hline $\mathrm{UA}, \mu \mathrm{mol} / \mathrm{L}$ & $369.2(298.3-450.0)$ & $381.4 \pm \mid 24.3$ & $368.0(300.0-446.2)$ & $394.2 \pm 130.4$ & 0.957 \\
\hline Glucose, mmol/L & $7.4(6.4-8.7)$ & $7.0(6.9-9.0)$ & $7.4(6.4-8.6)$ & $7.9 \pm 2.4$ & 0.817 \\
\hline BNP, pg/mL & $\begin{array}{c}472.9(189.5- \\
1160.5)\end{array}$ & $\begin{array}{c}596.7(109.0- \\
1445.5)\end{array}$ & $\begin{array}{c}442.0(163.0- \\
1133.0)\end{array}$ & $\begin{array}{c}592.0(376.0- \\
1169.0)\end{array}$ & 0.902 \\
\hline PT, s & $14.7(\mid 3.7-15.8)$ & $15.3 \pm 1.6$ & $14.6(13.6-15.5)$ & $15.3 \pm 1.4$ & 0.353 \\
\hline D-dimer, mg/L & $1.6(0.9-2.5)$ & $2.0 \pm 1.1$ & $1.6(0.9-2.3)$ & $1.9 \pm 1.3$ & 0.935 \\
\hline cTnl, ng/mL & $0.01(0.01-0.07)$ & $0.02(0.01-0.08)$ & $0.01(0.01-0.07)$ & $0.01(0.01-0.05)$ & 0.906 \\
\hline
\end{tabular}

Note: The italic and bold formatting indicates characteristics classification.

Abbreviations: HBP, high blood pressure; CAD, coronary artery disease; CKD, chronic kidney disease; DBP, diastolic blood pressure; WBC, white blood cell; Hb, hemoglobin; PLT, platelet; ALB, albumin; TB, total bilirubin; ALT, alanine transaminase; BUN, blood urea nitrogen; Cr, creatinine; UA, uric acid; BNP, brain natriuretic peptide; PT, prothrombin time; cTnl, cardiac troponin I.

\section{High Admission SBP in Patients with Anemia is an Effective Predictor of the Incidence of POD in AAAD Patients with}

\section{Surgical Repair}

In Table 1, hemoglobin showed no difference in SBP groups. Univariate analysis of anemia and POD revealed no difference, too (OR, 0.839; 95\% CI, 0.407-1.730; P > 0.05, Table 4). But subgroup analysis showed that patients with anemia and high admission SBP had a higher risk of POD (OR, 10.833; 95\% CI, 3.116-37.668; $\mathrm{P}<0.01$, interaction $\mathrm{P}$ value $<0.01$, Table 6). Similar results occurred in high urea nitrogen $(\mathrm{BUN})$ and creatinine $(\mathrm{Cr})$ subgroups. In patients with no HBP history, no anemia, age $<51$ years old, BUN $<7.1 \mathrm{mmol} / \mathrm{L}, \mathrm{Cr}<110 \mu \mathrm{mol} / \mathrm{L}$, high admission SBP level did not show increased risks of POD compared to normal admission SBP patient group (Table 6). 
Table 2 Treatments Between SBP Groups

\begin{tabular}{|c|c|c|c|c|c|}
\hline \multirow[t]{2}{*}{ Variables } & \multirow[t]{2}{*}{ Total $(n=205)$} & \multicolumn{3}{|c|}{ Blood Pressure $(\mathrm{mmHg})$} & \multirow[t]{2}{*}{$P$-value } \\
\hline & & $\leq 90(n=14)$ & $91-139(n=162)$ & $\geq 140(n=29)$ & \\
\hline Surgical procedure & & & & & 0.066 \\
\hline Sun's & I 30 (63.7\%) & $5(35.7 \%)$ & $108(67.1 \%)$ & $17(58.6 \%)$ & \\
\hline Sun's + root & $42(20.6 \%)$ & $6(42.9 \%)$ & $30(18.6 \%)$ & $6(20.7 \%)$ & \\
\hline Ascending + arch & $24(11.8 \%)$ & $3(21.4 \%)$ & $15(9.3 \%)$ & $6(20.7 \%)$ & \\
\hline Bentall & $8(3.9 \%)$ & $0(0.0 \%)$ & $8(5.0 \%)$ & $0(0.0 \%)$ & \\
\hline Operation duration, hours & $7.6(6.5-9.0)$ & $7.7 \pm 1.4$ & $7.5(6.5-8.9)$ & $8.0 \pm 2.2$ & 0.887 \\
\hline Aortic cross-clamp time, min & $105.0(80.0-124.0)$ & $122.5(110.0-128.0)$ & $103.0(80.0-120.0)$ & $115.4 \pm 44.7$ & 0.085 \\
\hline Cardiopulmonary bypass time, $\min$ & $187.0(\mid 66.5-210.5)$ & $187.7 \pm 29.3$ & $187.0(|66.0-2| 1.0)$ & $199.6 \pm 45.7$ & 0.736 \\
\hline Circulatory arrest time, min & $25.0(21.0-29.5)$ & $22.7 \pm 9.8$ & $25.0(21.0-29.0)$ & $26.0(22.5-32.0)$ & 0.281 \\
\hline \multicolumn{6}{|l|}{ Length of stay } \\
\hline Hospital stay, days & $16.0(\mid 2.0-21.5)$ & I5.5 (|I.0-2I.0) & $16.0(\mid 1.5-21.0)$ & $16.0(12.0-25.0)$ & 0.734 \\
\hline ICU stay, hours & $111.8(81.5-174.0)$ & $120.0(\mid 10.0-155.0)$ & I09.5 (77.0-184.0) & $128.5(96.5-161.0)$ & 0.444 \\
\hline
\end{tabular}

Notes: Sun's procedure indicates total arch replacement combined with stented elephant trunk implantation. Sun's + root indicates Sun's and aortic root replacement. Ascending + arch indicates ascending aorta and total arch replacement. Bentall indicates compound replacement of aortic root, aortic valve and ascending aorta, with reinsertion of coronary arteries into the new attachment or graft. ICU indicates intensive care unit. The italic and bold formatting indicates different variables.

Table 3 Outcomes Difference Between SBP Groups

\begin{tabular}{|c|c|c|c|c|c|}
\hline \multirow[t]{2}{*}{ Outcomes } & \multirow[t]{2}{*}{ Total $(n=205)$} & \multicolumn{3}{|c|}{ Blood Pressure (mmHg) } & \multirow[t]{2}{*}{$P$-value } \\
\hline & & $\leq 90(n=14)$ & $91-139(n=162)$ & $\geq 140(n=29)$ & \\
\hline Delirium & $36(17.6 \%)$ & I (7.I\%) & 24 (I4.8\%) & II (37.9\%) & 0.012 \\
\hline 30-day mortality & 29 (I4.1\%) & $2(14.3 \%)$ & $23(14.2 \%)$ & $4(13.8 \%)$ & 1.000 \\
\hline Acute kidney injury & $28(13.7 \%)$ & I (7.I\%) & $26(16.0 \%)$ & I (3.4\%) & 0.161 \\
\hline Cardiac complication & $22(10.7 \%)$ & $0(0.0 \%)$ & $19(11.7 \%)$ & $3(10.3 \%)$ & 0.575 \\
\hline Spinal cord ischemia & $14(6.8 \%)$ & $3(21.4 \%)$ & $9(5.6 \%)$ & $2(6.9 \%)$ & 0.087 \\
\hline Postoperative Stroke & $6(2.9 \%)$ & I (7.I\%) & I (0.6\%) & $4(13.8 \%)$ & 0.002 \\
\hline Pneumonia & $29(14.1 \%)$ & $2(14.3 \%)$ & $22(13.6 \%)$ & 5 (I7.2\%) & 0.809 \\
\hline
\end{tabular}

\section{Discussion}

In this study, we demonstrated that ICU admission $\mathrm{SBP} \geq 140 \mathrm{mmHg}$ was an independent risk factor and predictor of POD in AAAD patients who underwent surgery. Patients with anemia and high SBP had an increased risk of developing POD.

POD is one of the common complications of major vascular surgery, especially after AAAD surgery. The incidence of POD after major vascular surgery is 5$39 \%{ }^{16}$ Several studies reported that the occurrence of POD was associated with increased in-hospital mortality, longer hospital stay, cognitive decline, and decline in activities of daily living. ${ }^{17,18}$ However, a systemic review screening 5545 patients did not demonstrate a significant independent association of delirium with mortality. ${ }^{19}$ A recent retrospective study enrolling 301 AAAD patients in ICU stay indicated a higher in-hospital mortality rate but with no significance $(6.85 \%$ vs $4.82 \%, P=0.502) .^{20}$ Similarly, we did not find a significant difference between POD and in-hospital 30 -day mortality $(11.1 \%$ vs $14.8 \%$, $P=0.565$, data not shown).

The pathophysiology of POD is complex, elusive, and multifactorial. In recent years, various studies have demonstrated the risk factors for predicting POD of AAAD patients. ${ }^{16,21-23}$ A multi-drug addicted patient suffered from heroin-withdrawal POD after urgent AAAD repair, indicating that drug withdrawal was an important risk factor of POD, though no patient was addicted to drugs in our data. ${ }^{24}$ However, the association between systolic blood pressure (SBP) and delirium in patients with AAAD in ICU remains unknown. In the present study, we found high SBP level was positively associated with the incidence of POD. Patients with high and normal SBP were more likely to have a history of hypertension, 
Table 4 Predictors of POD on Univariate Logistic Regression and Cox Proportional Hazards Analysis

\begin{tabular}{|c|c|c|c|c|}
\hline \multirow[t]{2}{*}{ Variable } & \multicolumn{2}{|c|}{ Logistic Regression } & \multicolumn{2}{|c|}{ Cox Proportional Hazards } \\
\hline & OR $(95 \% \mathrm{CI})$ & $P$ value & HR (95\% Cl) & $P$ value \\
\hline Age & $1.012(0.977-1.047)$ & 0.513 & $1.019(0.969-1.07 I)$ & 0.459 \\
\hline Male sex & $1.225(0.537-2.793)$ & 0.629 & $0.743(0.282-1.960)$ & 0.743 \\
\hline $\mathrm{HBP}$ & $0.972(0.435-2.173)$ & 0.946 & $1.007(0.402-2.519)$ & 0.989 \\
\hline CAD & $0.706(0.152-3.274)$ & 0.656 & $1.064(0.196-5.773)$ & 0.942 \\
\hline Valvular disease & $2.565(0.820-8.022)$ & 0.106 & $2.286(0.768-6.808)$ & 0.137 \\
\hline Diabetes & $0.454(0.056-3.665)$ & 0.459 & $0.422(0.054-3.319)$ & 0.412 \\
\hline CKD & $1.581(0.160-15.648)$ & 0.695 & $0.000(0.000)$ & 0.978 \\
\hline Stroke/TIA & $0.816(0.284-2.340)$ & 0.705 & $0.756(0.239-2.393)$ & 0.635 \\
\hline Marfan's syndrome & $0.508(0.062-4.140)$ & 0.527 & $1.384(0.140-13.632)$ & 0.781 \\
\hline Anemia & $0.839(0.407-1.730)$ & 0.635 & $0.920(0.395-2.142)$ & 0.846 \\
\hline Aortic cross-clamp time & 1.001 (0.992-1.010) & 0.877 & $0.989(0.976-1.002)$ & 0.103 \\
\hline CPB time & $1.005(0.999-1.010)$ & 0.092 & $1.009(1.001-1.017)$ & 0.027 \\
\hline Circulatory arrest time & $1.013(0.970-1.058)$ & 0.554 & $0.989(0.976-1.002)$ & 0.989 \\
\hline Postoperative ventilation duration & $1.000(0.996-1.004)$ & 0.882 & $1.000(0.996-1.003)$ & 0.893 \\
\hline SBP & $1.026(1.007-1.046)$ & 0.004 & $3.064(1.311-7.158)$ & 0.010 \\
\hline DBP & $1.018(0.990-1.046)$ & 0.215 & $1.009(0.980-1.039)$ & 0.567 \\
\hline ALB & $1.060(0.980-1.146)$ & 0.147 & $1.069(0.978-1.169)$ & 0.140 \\
\hline TB & $1.026(0.990-1.063)$ & 0.162 & $1.028(0.993-1.063)$ & 0.115 \\
\hline $\mathrm{Cr}$ & 1.001 (0.997-1.006) & 0.507 & $1.001(0.993-1.010)$ & 0.747 \\
\hline PT & 1.091 (0.976-1.219) & 0.125 & $1.048(0.959-1.145)$ & 0.303 \\
\hline D-Dimer & $1.018(0.908-1.140)$ & 0.761 & $1.038(0.935-1.152)$ & 0.488 \\
\hline
\end{tabular}

Abbreviations: HBP, high blood pressure; CAD, coronary artery disease; CKD, chronic kidney disease; CPB, cardiopulmonary bypass; SBP, systolic blood pressure; DBP, diastolic blood pressure; ALB, albumin; TB, total bilirubin; Cr, creatinine; PT, prothrombin time.

Table 5 ORs (95\% Cls) for Delirium Across Groups of Admission Systolic Blood Pressure

\begin{tabular}{|l|c|c|c|c|}
\hline \multirow{2}{*}{ SBP (mmHg) } & \multicolumn{2}{|c|}{ Non-Adjusted } & \multicolumn{2}{c|}{ Model } \\
\cline { 2 - 5 } & OR (95\% Cl) & P value & OR (95\% Cl) & P value \\
\hline$\leq 90$ & $0.442(0.055-3.539)$ & 0.442 & $0.621(0.070-5.518)$ & 0.669 \\
$91-139$ & $1.0($ ref $)$ & $/$ & $1.0($ ref $)$ & $/$ \\
$\geq 140$ & $3.514(1.478-8.357)$ & 0.004 & $4.655(1.708-12.687)$ & 0.003 \\
\hline
\end{tabular}

Notes: ORs were calculated from logistic regression models. Non-adjusted model adjusted to none. Model adjusted to demographics, history of diabetes, coronary artery disease, cardiac valvular disease, Marfan's syndrome, chronic kidney injury, cardiopulmonary bypass time, aortic cross-clamp time, hemoglobin, albumin, total bilirubin, alanine transaminase, creatinine, uric acid, and D-dimer.

Abbreviations: SBP, systolic blood pressure; OR, odds ratio; ref, reference.

higher DBP, and higher mean arterial pressure, which is properly in line with clinical observations. Univariate analysis and Cox proportional hazard model showed that high SBP was a predictor of POD. Furthermore, multivariate logistic regression analysis after adjustment for demographics, medical history, operation variables, and laboratory tests, high SBP still showed a positive association with POD occurrence. While low admission SBP level did not show relevance to POD. These results collectively demonstrated that high admission SBP level was an independent risk factor and predictor of POD in patients with surgical repair.
Previous studies showed hypertension was a risk factor of POD after cardiac and vascular surgery. ${ }^{7,8,22}$ There are several controversial studies regarding different blood pressure indexes (not SBP) as predictors of POD. A prospective cohort study enrolling 2914 patients established a prediction model of delirium, consisting of mean arterial blood pressure (MAP). ${ }^{25}$ Echigoya et al found that permissive hypotension was a risk factor of POD after abdominal surgery in elderly patients. ${ }^{26}$ Hirsch et al reported that increased blood pressure fluctuation, not absolute or relative hypotension, was predictive of POD. ${ }^{27}$ Contrary to these studies, our results showed that 
Table 6 Subgroup Analysis of the Association Between Systolic Blood Pressure and Delirium

\begin{tabular}{|c|c|c|c|c|c|}
\hline & \multirow[t]{2}{*}{ Num of Patients } & \multicolumn{3}{|c|}{ Admission Systolic Blood Pressure (mmHg) } & \multirow[t]{2}{*}{$P$-value for Interaction } \\
\hline & & $\leq \mathbf{9 0}$ & $91-139$ & $\geq 140$ & \\
\hline Overall population & 205 & $0.442(0.055-3.539)$ & Ref & $3.514(1.478-8.357)$ & \\
\hline Age, years & & & & & 0.057 \\
\hline$<51$ & 102 & $0.000(0.000)$ & Ref & $2.939(0.758-11.397)$ & \\
\hline$\geq 51$ & 103 & $0.825(0.093-7.318)$ & Ref & $4.200(1.320-13.367)$ & \\
\hline Gender & & & & & 0.213 \\
\hline Male & 147 & $0.759(0.088-6.531)$ & Ref & $3.101(1.082-8.889)$ & \\
\hline Female & 58 & $0.000(0.000)$ & Ref & $4.933(1.024-23.772)$ & \\
\hline HBP & & & & & 0.044 \\
\hline No & 56 & $0.000(0.000)$ & Ref & $2.829(0.545-14.690)$ & \\
\hline Yes & 149 & $1.235(0.136-\mid 1.231)$ & Ref & $3.80 \mathrm{I}(1.372-10.529)$ & \\
\hline Anemia & & & & & 0.003 \\
\hline No & 106 & $0.000(0.000)$ & Ref & $0.889(0.176-4.478)$ & \\
\hline Yes & 99 & $1.354(0.144-12.730)$ & Ref & $10.833(3.116-37.668)$ & \\
\hline Hyperuricemia & & & & & 0.025 \\
\hline No & 138 & $0.000(0.000)$ & Ref & 3.231 (1.057-9.879) & \\
\hline Yes & 67 & $0.606(0.066-5.578)$ & Ref & $4.545(1.041-19.857)$ & \\
\hline BUN, mmol/L & & & & & 0.014 \\
\hline$<7.1$ & 107 & $0.948(0.106-8.463)$ & Ref & $2.413(0.652-8.928)$ & \\
\hline$\geq 7.1$ & 97 & $0.000(0.000)$ & Ref & $4.923(1.475-16.434)$ & \\
\hline $\mathrm{Cr}, \mu \mathrm{mol} / \mathrm{L}$ & & & & & 0.007 \\
\hline$<110$ & 114 & $0.000(0.000)$ & Ref & $1.974(0.552-7.066)$ & \\
\hline$\geq 110$ & 89 & $1.073(0.114-10.091)$ & Ref & $6.258(1.764-22.199)$ & \\
\hline
\end{tabular}

Notes: Anemia was diagnosed by hemoglobin cut-off level below $110 \mathrm{~g} / \mathrm{L}$ for females and below $120 \mathrm{~g} / \mathrm{L}$ for males. Hyperuricemia was defined as uric acid $>428.0 \mu$ mol/L in males and $>357.0 \mu \mathrm{mol} / \mathrm{L}$ in females. The bold formatting indicates subgroup grouping basis.

Abbreviations: BUN, blood urea nitrogen; $\mathrm{Cr}$, creatinine; ref, reference.

none of the histories of hypertension, MAP, or DBP was associated with POD. Reasons for inconsistent results include the differences in research design, population, and sample size.

The underlying mechanisms between hypertension and neurological dysfunction include abnormal cerebral blood flow, dysfunctional autoregulation, metabolic, cellular, neurochemical disturbances, or psychophysiological reactivity. ${ }^{28}$ Increased blood pressure under conditions of impaired autoregulation increases cerebral blood flow, leading to transudation of fluid into the pericapillary astrocytes and interstitium of the brain. ${ }^{29}$ SBP above the threshold of autoregulation contributes to an increase in cerebral blood flow, resulting in excessive cerebral microembolic load, endothelial damage, and compromise to the bloodbrain barrier. These aberrations expose patients to neuroinflammation, which has been suggested to contribute to delirium susceptibility. ${ }^{30,31}$ Shi et al clarified that antegrade selective cerebral perfusion time was an independent factor for POD of AAAD, for the reason that cerebral blood flow was more dependent on selective cerebral perfusion pressure in patients with hypertension. ${ }^{22}$ Collectively, the management of SBP was important to reduce the occurrence of POD.

Subgroup analysis showed that regardless of whether patients were grouped by gender or hyperuricemia, high admission SBP was a risk factor of POD. In subgroups of anemia, age $\geq 51$ years old, $\mathrm{BUN} \geq 7.1 \mathrm{mmol} / \mathrm{L}$, and $\mathrm{Cr}$ $\geq 110 \mu \mathrm{mol} / \mathrm{L}$, patients with high admission SBP were at higher risk of POD. However, in subgroups of nonanemia, age $<51$ years old, BUN $<7.1 \mathrm{mmol} / \mathrm{L}$, and $\mathrm{Cr}$ $<110 \mu \mathrm{mol} / \mathrm{L}$, high SBP was not a risk factor. Regardless of the imbalance between subgroups, these results agreed with several prospective cohort studies..$^{25,32,33}$ In the IPDACS (Incidence and Predictors of Delirium After Cardiac Surgery) study which recruited 563 patients, 
Kazmierski et al reported that anemia was independently associated with delirium after cardiac surgery. ${ }^{34}$ Granberg and Kwizera reported that mechanically ventilated patients who developed delirium are more likely to have decreased hemoglobin concentration. ${ }^{35,36}$ Kunz et al reported that $38.4 \%$ of patients with postoperative anemia suffered from POD (OR 3.949, 1.358-11.480). ${ }^{37}$ Since anemia may be an aggressive risk factor of POD in AAAD patients with high SBP, erythrocyte transfusion could be a useful way to reduce the risk of POD. Vera Zanden et al reported that, in 415 patients with hip fracture surgery, anemia (hemoglobin level $\leq 9.7 \mathrm{~g} / \mathrm{dl}$ ) was associated with POD (OR 1.81, 1.15-2.86), and blood transfusion was a protective factor for POD in anemic patients. ${ }^{38}$ In contrast, Janssen et al reported that erythrocyte transfusion was a risk factor for POD in elderly patients with major abdominal surgery. ${ }^{21}$ One possible mechanism underlying anemia and POD is chronic hypoperfusion of brain regions including the periventricular white matter, basal ganglia, and hippocampus, leading to cognitive disorders. ${ }^{39}$ Unfortunately, we did not collect data on brain malperfusion represented by Penn classification, which might be a significant confounding factor. ${ }^{40}$

Several limitations of the present study should be noted. First, this retrospective study was conducted in a single center with a relatively small sample size, for which biases could not be completely avoided, though we have used consecutive selection, review of electronic medical records, and multivariate logistic analysis to minimize the biases. Second, blood pressure is a variable that can be affected by many factors, it would be more reasonable to enroll in preoperative, intraoperative blood pressure excursion to investigate perioperative risk factors of POD. However, since systolic blood pressure in ICU was controlled between 100 and $120 \mathrm{mmHg}$, we emphasize that admission SBP may reflect the actual condition of the acute aortic syndrome. Third, assessing delirium 3 times a day would be inadequate to discover the transient POD cases and bias the results. It could be more reasonable to assess POD every 4-8 hours to avoid the loss of positive cases. Furthermore, in-depth mechanism exploration, clinical data, and prospective studies are expected to confirm our findings.

\section{Conclusion}

This study confirmed that high admission SBP was positively associated with the incidence of POD. Optimizing the admission SBP might provide a strategy for reducing the risk of POD in AAAD patients with surgical repair.

\section{Disclosure}

The authors report no conflicts of interest in this work.

\section{References}

1. Pape LA, Awais M, Woznicki EM, et al. Presentation, diagnosis, and outcomes of acute aortic dissection: 17-year trends from the international registry of acute aortic dissection. $J$ Am Coll Cardiol. 2015;66 (4):350-358. doi:10.1016/j.jacc.2015.05.029

2. Wilson JE, Mart MF, Cunningham C, et al. Delirium. Nat Rev Dis Primers. 2020;6(1):90.

3. Whitlock EL, Vannucci A, Avidan MS. Postoperative delirium Minerva Anestesiol. 2011;77(4):448-456.

4. Bossone E, Gorla R, LaBounty TM, et al. Presenting systolic blood pressure and outcomes in patients with acute aortic dissection. $J \mathrm{Am}$ Coll Cardiol. 2018;71(13):1432-1440. doi:10.1016/j. jacc.2018.01.064

5. Wahlsten LR, Zareini B, Smedegaard L, et al. A medical history of arterial thrombosis is a strong predictor of post-operative myocardial infarction and stroke in patients with hip fractures - a nationwide cohort study. Age Ageing. 2021;50(4):1252-1260. doi:10.1093/ageing/afaa279

6. Demir AR, Celik O, Uygur B, et al. Determination of cerebrovascular accident predictors in patients with new-onset atrial fibrillation after coronary artery bypass graft surgery. J Stroke Cerebrovasc Dis. 2021;30(3):105591. doi:10.1016/j.jstrokecerebrovasdis.2020.105591

7. Yang Q, Wang J, Huang X, et al. Incidence and risk factors associated with postoperative delirium following primary elective total hip arthroplasty: a retrospective nationwide inpatient sample database study. BMC Psychiatry. 2020;20(1):343. doi:10.1186/s12888-02002742-6

8. Oliveira FR, Oliveira VH, Oliveira M, et al. Hypertension, mitral valve disease, atrial fibrillation and low education level predict delirium and worst outcome after cardiac surgery in older adults. $B M C$ Anesthesiol. 2018;18(1):15. doi:10.1186/s12871-018-0481-0

9. Ely EW, Margolin R, Francis J, et al. Evaluation of delirium in critically ill patients: validation of the Confusion Assessment Method for the Intensive Care Unit (CAM-ICU). Crit Care Med. 2001;29(7):1370-1379. doi:10.1097/00003246-200107000-00012

10. Palevsky PM, Liu KD, Brophy PD, et al. KDOQI US commentary on the 2012 KDIGO clinical practice guideline for acute kidney injury. Am J Kidney Dis. 2013;61(5):649-672. doi:10.1053/j. ajkd.2013.02.349

11. Romi F, Naess H. Spinal cord infarction in clinical neurology: a review of characteristics and long-term prognosis in comparison to cerebral infarction. Eur Neurol. 2016;76(3-4):95-98. doi:10.1159/ 000446700

12. Bijker JB, Persoon S, Peelen LM, et al. Intraoperative hypotension and perioperative ischemic stroke after general surgery: a nested case-control study. Anesthesiology. 2012;116(3):658-664. doi:10.1097/ALN.0b013e3182472320

13. Chughtai M, Gwam CU, Mohamed N, et al. The epidemiology and risk factors for postoperative pneumonia. J Clin Med Res. 2017;9 (6):466-475. doi:10.14740/jocmr3002w

14. D'Agostino RS, Jacobs JP, Badhwar V, et al. The society of thoracic surgeons adult cardiac surgery database: 2017 update on outcomes and quality. Ann Thorac Surg. 2017;103(1):18-24. doi:10.1016/j. athoracsur.2016.11.001

15. World Medical Association. Declaration of Helsinki: ethical principles for medical research involving human subjects. JAMA. 2013;310 (20):2191-2194. doi:10.1001/jama.2013.281053 
16. Aitken SJ, Blyth FM, Naganathan V. Incidence, prognostic factors and impact of postoperative delirium after major vascular surgery: a meta-analysis and systematic review. Vasc Med. 2017;22(5):387-397. doi:10.1177/1358863X17721639

17. Shi Z, Mei X, Li C, et al. Postoperative delirium is associated with long-term decline in activities of daily living. Anesthesiology. 2019;131(3):492-500. doi:10.1097/ALN.0000000000002849

18. Goldberg TE, Chen C, Wang Y, et al. Association of delirium with long-term cognitive decline: a meta-analysis. JAMA Neurol. 2020;77 (11):1-9. doi:10.1001/jamaneurol.2020.2273

19. Nienaber CA, Clough RE, Sakalihasan N, et al. Aortic dissection. Nat Rev Dis Primers. 2016;2:16053. doi:10.1038/nrdp.2016.53

20. Cai S, Zhang X, Pan W, et al. Prevalence, predictors, and early outcomes of post-operative delirium in patients with type A aortic dissection during intensive care unit stay. Front Med. 2020;7:572581. doi:10.3389/fmed.2020.572581

21. Janssen TL, Steyerberg EW, Faes MC, et al. Risk factors for postoperative delirium after elective major abdominal surgery in elderly patients: a cohort study. Int J Surg. 2019;71:29-35. doi:10.1016/j. ijsu.2019.09.011

22. Shi Q, Mu X, Zhang C, et al. Risk factors for postoperative delirium in type A aortic dissection patients: a retrospective study. Med Sci Monit. 2019;25:3692-3699. doi:10.12659/MSM.913774

23. Galyfos GC, Geropapas GE, Sianou A, et al. Risk factors for postoperative delirium in patients undergoing vascular surgery. $J$ Vasc Surg. 2017;66(3):937-946. doi:10.1016/j.jvs.2017.03.439

24. Gaetano R, De Filippis R, Caroleo M, et al. Postoperative heroin-withdrawal delirium treated with clonazepam after urgent cardiac surgery: a case report. Riv Psichiatr. 2020;55(6):366-370.

25. Wassenaar A, van den Boogaard $M$, van Achterberg $T$, et al. Multinational development and validation of an early prediction model for delirium in ICU patients. Intensive Care Med. 2015;41 (6):1048-1056. doi:10.1007/s00134-015-3777-2

26. Echigoya Y, Kato H. [Causes of postoperative delirium after abdominal surgery in elderly patients]. Masui. 2007;56(8):932-936. Japanese.

27. Hirsch J, DePalma G, Tsai TT, et al. Impact of intraoperative hypotension and blood pressure fluctuations on early postoperative delirium after non-cardiac surgery. Br J Anaesth. 2015;115(3):418-426. doi:10.1093/bja/aeu458

28. Waldstein SR, Manuck SB, Ryan CM, et al. Neuropsychological correlates of hypertension: review and methodologic considerations. Psychol Bull. 1991;110(3):451-468. doi:10.1037/00332909.110.3.451
29. Schwartz RB. Hyperperfusion encephalopathies: hypertensive encephalopathy and related conditions. Neurologist. 2002;8(1):22-34. doi:10.1097/00127893-200201000-00003

30. Inouye SK. Delirium in older persons. $N$ Engl J Med. 2006;354 (11):1157-1165. doi:10.1056/NEJMra052321

31. Aggarwal M, Khan IA. Hypertensive crisis: hypertensive emergencies and urgencies. Cardiol Clin. 2006;24(1):135-146. doi:10.1016/j. ccl.2005.09.002

32. Kubota K, Suzuki A, Ohde S, et al. Age is the most significantly associated risk factor with the development of delirium in patients hospitalized for more than five days in surgical wards: retrospective cohort study. Ann Surg. 2018;267(5):874-877. doi:10.1097/ SLA.0000000000002347

33. Kalisvaart KJ, Vreeswijk R, de Jonghe JFM, et al. Risk factors and prediction of postoperative delirium in elderly hip-surgery patients: implementation and validation of a medical risk factor model. $J \mathrm{Am}$ Geriatr Soc. 2006;54(5):418-426. doi:10.1111/j.15325415.2006.00704.x

34. Kazmierski J, Kowman M, Banach M, et al. Incidence and predictors of delirium after cardiac surgery: results from The IPDACS Study. J Psychosom Res. 2010;69(2):179-185. doi:10.1016/j. jpsychores.2010.02.009

35. Granberg Axèll AI, Malmros CW, Bergbom IL, et al. Intensive care unit syndrome/delirium is associated with anemia, drug therapy and duration of ventilation treatment. Acta Anaesthesiol Scand. 2002;46 (6):726-731. doi:10.1034/j.1399-6576.2002.460616.x

36. Kwizera A, Nakibuuka J, Ssemogerere L, et al. Incidence and risk factors for delirium among mechanically ventilated patients in an African intensive care setting: an observational multicenter study. Crit Care Res Pract. 2015;2015:491780.

37. Kunz JV, Spies CD, Bichmann A, et al. Postoperative anaemia might be a risk factor for postoperative delirium and prolonged hospital stay: a secondary analysis of a prospective cohort study. PLoS One. 2020;15(2):e0229325. doi:10.1371/journal.pone.0229325

38. van der Zanden V, Beishuizen SJ, Scholtens RM, et al. The effects of blood transfusion on delirium incidence. $J$ Am Med Dir Assoc. 2016;17(8):748-753. doi:10.1016/j.jamda.2016.04.008

39. Román GC. Brain hypoperfusion: a critical factor in vascular dementia. Neurol Res. 2004;26(5):454-458. doi:10.1179/ 016164104225017686

40. Tien M, Ku A, Martinez-Acero N, et al. The Penn classification predicts hospital mortality in acute Stanford type A and type B aortic dissections. J Cardiothorac Vasc Anesth. 2020;34 (4):867-873. doi:10.1053/j.jvca.2019.08.036
International Journal of General Medicine

\section{Publish your work in this journal}

The International Journal of General Medicine is an international, peer-reviewed open-access journal that focuses on general and internal medicine, pathogenesis, epidemiology, diagnosis, monitoring and treatment protocols. The journal is characterized by the rapid reporting of reviews, original research and clinical studies across all disease areas. The manuscript management system is completely online and includes a very quick and fair peer-review system, which is all easy to use. Visit http://www.dovepress.com/ testimonials.php to read real quotes from published authors. 